\title{
MITOS DA DIDÁTICA DAS CIÊNCIAS ACERCA DOS MOTIVOS PARA INCLUIR A NATUREZA DA CIÊNCIA NO ENSINO DAS CIÊNCIAS
}

\author{
Science Education myths about the reasons to include the \\ Nature of Science in science teaching
}

\author{
Acevedo, J. A. ${ }^{1}$ \\ Vázquez, $A .^{2}$ \\ Paixāo, M. F. ${ }^{3}$ \\ Acevedo, P.4 \\ Oliva J. M.5 \\ Manassero, $M .{ }^{6}{ }^{6}$
}

\begin{abstract}
Resumo: Em certas situações, a Didática das Ciências transmite como mitos algumas crenças que não estão suficientemente sustentadas pela investigação que ela própria produz. Este artigo mostra dois desses mitos relacionados com os motivos que se costumam apontar para incluir a Natureza da Ciência no ensino das ciências, como sejam a suposta relação entre a prática docente e as crenças sobre a Natureza da Ciência, e a crença de que a sua compreensão é um fator chave na hora de tomar melhores decisões cívicas em questôes tecnocientíficas de interesse social. A análise que se apresenta realizou-se mediante a revisão de diversos resultados de investigaçôes procedentes da própria Didática das Ciências e também da Psicologia das Decisões. A conclusão aponta para considerar que outros fatores influenciam mais, tornando muito menos lineares essas hipotéticas relaçóes do que alguns especialistas pensam e mais complexa a problemática abordada.
\end{abstract}

Unitermos: Natureza da Ciência; Didática das Ciências; ensino das ciências; prática docente; decisões tecnocientíficas.

Abstract: In some situations Science Education transmits as myths various beliefs as myths that are not enough sustained by the research in its own domain. This paper shows two of these myths related to the reasons usually pointed in orderfor includinge the Nature of Science in science teaching:, such as the supposed relationship between the educational practice and the beliefs about the Nature of Science, and also the belief that its understanding of the Nature of Science is a key factor in when making better civicdecisions as citizens in technical socioscientific oscientific issues. with social interest The analysis was carried out by means of athe review of several results from the own Science Education Research, and also from the Psychology of Decisions. The conclusion seems to be clear: other factors are of a greater influence, making those hypothetical relations much less linear than some experts wcould think, and making the questions much more complex. the presented question.

Keywords: Nature of Science; Science Education; science teaching; educational practice; technoscientific decisions.

\footnotetext{
${ }^{1}$ Inspetor de Educação. Consejería de Educación de la Junta de Andalucía. Delegación Provincial de Huelva-España. E-mail: ja_acevedo@vodafone.es

${ }^{2}$ Inspetor de Educação e Professor Associado. Universidad de las Islas Baleares-España.

E-mail:avazquez@dgadmedu.caib.es

${ }^{3}$ Professora Coordenadora. Escola Superior de Educação, Instituto Politécnico de Castelo Branco-Portugal.

E-mail:fatimapaixao@ese.ipcb.pt

${ }^{4}$ Colaboradora de Investigação. Universidad de Sevilla-España.E-mail: pi_acevedo@yahoo.es

${ }^{5}$ Professor Catedrático de Física e Química do Ensino Secundário. Centro de Profesorado de Cádiz-España.

E-mail: jmoliva@cepcadiz.com

${ }^{6}$ Professora Catedrática. Universidad de las Islas Baleares-España. E-mail: ma.manassero@uib.es
} 


\section{Introdução}

É cada vez maior, em didática das ciências, o consenso de considerar que um dos objetivos mais importantes da educação científica é que os estudantes do Ensino Básico e do Secundário cheguem a adquirir uma melhor compreensão da natureza da ciência $-\mathrm{NdC}-$ (Matthews, 1998b; McComas, Clough e Almazroa, 1998). Deste modo, a presença da NdC no currículo de ciências é valorizada pelos que concebem uma educação científica mais apropriada para o século XXI. Hoje em dia vários países - por exemplo, Austrália, Canadá, Inglaterra, Nova Zelândia, USA etc. - incluem explicitamente o ensino da NdC nos seus currículos científicos das suas reformas (AAAS, 1989; 1993; Department for Education and Employment, 1999; McComas e Olson, 1998; NRC, 1996) e muitos outros o fazem de uma forma mais ou menos parcial e mais ou menos implícita.

Apesar de este objetivo ser bastante antigo e se renovar periodicamente na bibliografia especializada, as razões que se têm dado para o implementar tendem a alterar-se com o tempo (Lederman, 1992). Assim, nos últimos anos apareceu como um dos componentes essenciais da alfabetização científica e tecnológica para todas as pessoas (AAAS, 1989, 1993; Bybee, 1997; Department for Education and Employment, 1999; NRC, 1996; Reid e Hodson, 1989) e da educação CTS (Acevedo, 1994, 1996b; Acevedo, Vázquez e Manassero, 2002; Caamaño, 1996; Spector, Strong e Laporta, 1998). Em suma, como assinalou Hodson (1994), pensa-se que um dos principais objetivos do ensino das ciências é a aprendizagem da $\mathrm{NdC}$, tanto para desenvolver uma melhor compreensão da ciência e seus métodos como para contribuir para tomar mais consciência das interaçôes entre a ciência, a tecnologia e a sociedade.

O conhecimento da $\mathrm{NdC}$ é em grande parte um metaconhecimento que surge da reflexão sobre a própria ciência, pelo que poderia parecer um objetivo pouco razoável. Sendo que a metacognição constitui o nível de maior complexidade no desenvolvimento cognitivo humano, a compreensão da $\mathrm{NdC}$ poderia ficar de fora do alcance da grande maioria dos alunos. Por outro lado, também se tem apontado como dificuldade importante para estabelecer que conteúdos de $\mathrm{NdC}$ se devem ensinar, o fato de que os próprios filósofos e sociólogos da ciência têm grandes desacordos sobre os princípios básicos desta (Alters, 1997; Vázquez et al., 2001), devido ao caráter dialético e controverso das questôes postas em jogo e também da maior tendência destes profissionais para a polêmica. Além disso, parte dessas visōes, também não coincidem com algumas das que se defendem a partir do campo da própria ciência; recorde-se a denominada "guerra das ciências" como uma virulenta reação dos cientistas aos excessos dos relativistas e socioconstrutivistas pós-modernos mais radicais (Gross e Levitt, 1994; Sokal e Bricmont, 1998; Wolpert, 1992). Contudo, a maioria destas discrepâncias referem-se a questôes demasiado abstratas para terem alguma repercussão na vida diária dos alunos e na cidadania (Abd-El-Khalick e Boujaoude, 1997), pelo que não se deve pretender reproduzir na escola este tipo de reflexão metacognitiva, nem tão pouco entrar nos complexos problemas que ainda estão por resolver; o objetivo a perseguir não é formar filósofos nem sociólogos da ciência, mas ajudar a compreender melhor a ciência e a tecnologia contemporâneas. Talvez possa ser possível conseguir algum consenso (Abd-El-Khalick, Bell e Lederman, 1998; Eflin, Glennan e Reisch, 1999; Felske, Chiappetta e Kemper, 2001; Manassero, Vázquez e Acevedo, 2004; McComas e Olson, 1998; Osborne et al., 2001, 2003; Smith et al., 1997; Vázquez, Acevedo e Manassero, 2004; Vázquez et al., 2001, 2004), que possa ser a base para definir objetivos e conteúdos mais modestos, mais adaptados ao nível de desenvolvimento dos alunos e mais ajustados aos requisitos de um ensino das ciências destinado a uma alfabetização científica e tecnológica para todos (Matthews, 1998a). 
De acordo com esta idéia, os métodos para ensinar NdC têm-se, recentemente, mostrado eficazes quando abordam alguns dos seus aspectos básicos de maneira explícita e reflexiva; ou seja, se tal se faz com uma boa planificação, desenvolvendo os conteúdos em atividades variadas e avaliando os processos desenvolvidos e os resultados conseguidos (Akerson, Abd-El-Khalick e Lederman, 2000). Têm-se relatado resultados positivos quando se usam atividades baseadas na pesquisa científica (Schwartz et al., 2002), na História e Filosofia da Ciência (Abd-ElKhalick, 2002; Abd-El-Khalick e Lederman, 2000b; Irwin, 2000; Lin e Chen, 2002; Rudolph, 2003), contextualizadas (Matkins et al., 2002) com um enfoque CTS do tipo IOS - IssueOriented-Science - (Acevedo e Acevedo, 2002; Acevedo, Vázquez e Manassero, 2002; Spector, Strong e Laporta, 1998) e capazes de relacionar o mundo real e quotidiano dos alunos (Khishfe e AbdEl-Khalick, 2002). Mesmo assim, têm sido conduzidos projetos expressamente concebidos para melhorar a compreensão da $\mathrm{NdC}$ que colocam o acento nos processos sociais da construção do conhecimento científico e na resolução das controvérsias científicas (Kolstø, 2000, 2001; Kolstø e Mestad, 2003; Kolstø et al., 2004). Estas linhas de trabalho puseram também em questão a crença de que um ensino implícito da $\mathrm{NdC}$, baseadoretudo na prática dos procedimentos da ciência e outros conteúdos indiretos, permite alcançar uma boa compreensão da NdC (Bell, Lederman e Abd-El-Khalick, 1998, 2000).

Os parágrafos anteriores deixam clara a atenção preferencial que a didática das ciências têm vindo prestar à $\mathrm{NdC}$ e o seu papel na reforma do ensino das ciências. Para isso, ao longo dos anos têm sido dados contributos e continuam a apresentar-se diversas razôes para motivar a importância que lhe tem sido concedida, sejam razões utilitárias, democráticas, culturais, axiológicas e relacionadas com o ensino e a aprendizagem dos conceitos científicos (Driver et al., 1996); argumentos que nem sempre estão apoiados pelos resultados das investigaçôes realizadas. Por exemplo, tem-se sustentado, e continua a apoiar-se acriticamente, que as crenças dos professores sobre a $\mathrm{NdC}$ se relacionam diretamente com a sua prática docente. Ao mesmo tempo, afirmou-se e continua a afirmar-se que uma boa compreensão da $\mathrm{NdC}$ se apresenta como um fator decisivo para tomar melhores decisões sobre questôes tecnocientíficas de interesse social. Em seguida, tratar-se-ão estes dois pressupostos da didática das ciências, para mostrar que esta cria, por vezes, os seus próprios mitos e os propaga, como sucede quando decide dar valor à $\mathrm{NdC}$ no ensino das ciências.

\section{Natureza da ciência e prática docente}

Desde a última década do século passado o estudo das crenças dos professores sobre a $\mathrm{NdC}$ se tornou um tema prioritário da investigação em didática das ciências, persistindo o seu interesse no presente (Adúriz-Bravo, 2001; Fernández-Montoro, 2000). Desde o primeiro momento, estas investigações basearam-se em duas hipóteses implícitas (Lederman, 1992):

1. A compreensão dos professores acerca da $\mathrm{NdC}$ tem uma certa relação com a dos seus alunos e com a imagem que estes adquirem da ciência.

2. As crenças dos professores sobre a $\mathrm{NdC}$ influenciam significativamente na sua forma de ensinar ciências e nas decisões que tomam na aula.

Contudo, por muito atrativas que possam parecer ambas as hipóteses, o certo é que ainda não estão suficientemente validadas pelas investigações realizadas (Canavarro, 2000; Carvajal e Gómez-Villarta, 2002; Duschl e Wright, 1989; Lederman, 1992, 1999; Lederman, McComas e Matthews, 1998; McComas, Clough e Almazroa, 1998), algumas das quais, 
inclusive, proporcionam dados contra (Lederman e Zeidler, 1987; Mellado, 1996, 1997, 1998). Num trabalho recente, Tsai (2002) apresentou resultados que mostram uma importante relação entre as crenças dos professores em relação ao ensino, à aprendizagem e à $\mathrm{NdC}$; contudo, como adverte o próprio autor do artigo, isso não significa necessariamente que também exista coerência com a prática docente, um aspecto que não foi objeto dessa investigação.

Por outro lado, diversos investigadores têm assinalado vários fatores que influem quando o professor transfere para a aula conteúdos de NdC (Abd-El-Khalick e Lederman, 2000a; Bartholomew, Osborne e Ratcliffe, 2002; Lederman e Zeidler, 1987; Mellado, 1996, 1997, 1998; Schwartz e Lederman, 2002). A maioria desses fatores não tem a ver com os próprios conteúdos de $\mathrm{NdC}$, mas sim com resistências gerais às inovaçōes educativas e, principalmente, com o conhecimento didático do conteúdo - pedagogical content knowledge - (Gess-Newsome e Lederman, 1999), uma noção introduzida por Shulman $(1986,1987)$ para expressar o conhecimento profissional específico que os professores desenvolvem sobre a forma de ensinar a sua disciplina e que é, afinal, a intersecção entre os conhecimentos didáticos, do tema e do objeto de ensino - a $\mathrm{NdC}$, neste caso -, que também se relaciona com a necessária transposição didática dos conteúdos que devem transferir para a aula. Sem dúvida, estes aspectos adicionam muito mais complexidade ao que se sustenta linearmente nas duas hipóteses indicadas.

Apesar de tudo isto, estas hipóteses têm sido assumidas de maneira acrítica em muitos desenhos curriculares das reformas empreendidas na década de 1990. Por exemplo, na introdução do currículo da área de Ciências da Natureza da Educação Secundária Obrigatória da Comunidade Autônoma da Andaluzia (Espanha) afirmava-se que:

\section{[...] Existe una estrecha relación entre la concepción que sobre la naturaleza de la ciencia se posee $y$ los tipos de aprendizajes que se promueven en los alumnos.}

[Decreto 106/1992, anexo II, p. 4126. (CECJA, 1992)]

Este parágrafo volta a ser reproduzido literalmente, dez anos depois, na introdução do novo currículo de Ciências da Natureza da Educação Secundária Obrigatória estabelecido para a Comunidade Autônoma da Andaluzia (CECJA, 2002). Parece, pois, que a normativa legal ignora, neste caso, os contributos da investigação internacional sobre este tema ou então que está enviesada pelas crenças não provadas mas sustentadas por alguns especialistas em didática das ciências.

\section{Natureza da ciência e tomada de decisóes pelos cidadãos}

Como se referiu na introdução, o ensino da $\mathrm{NdC}$ aparece cada vez mais associado à alfabetização científica e tecnológica de todas as pessoas. Para isso, numerosos especialistas em didática das ciências têm apelado a um argumento democrático, ou seja, no sentido de que uma melhor compreensão da $\mathrm{NdC}$ permite tomar decisōes mais refletidas sobre questões tecnocientíficas de interesse social, o que contribuiria para tornar mais possível a participação na cidadania (Driver et al., 1996). Na mesma linha, Abd-El-Khalick (2001) sugeriu que as decisões dos estudantes em assuntos sociocientíficos são análogas às que têm que tomar os cientistas para justificar o conhecimento que geram; em ambos os casos se requer um discurso racional e, ao mesmo tempo, senso comum e capacidade para valorar os argumentos. Mas, é o conhecimento da $\mathrm{NdC}$ um fator chave para tomar este tipo de decisão?

Num trabalho em que se investigou a relação entre as concepções sobre a $\mathrm{NdC}$ de estudantes do Secundário e da Universidade e as suas reaçôes perante provas científicas que desafiavam as suas crenças relativamente a diversas questôes sociocientíficas, Zeidler et al. 
(2002) mostraram que muitos deles consideraram irrelevante para tomar as suas decisões qualquer conhecimento científico que não apoie as suas crenças prévias; de outro modo, à margem do mérito científico dos dados que lhes proporcionaram, os estudantes tendem a selecionar a informação que está mais de acordo com as suas crenças pessoais sobre o tema colocado. Ainda que muitos deles aceitaram os dados científicos oferecidos, depois preferiram não os usar nos pensamentos que seguiram para tomar as suas decisões sobre as questóes sociocientíficas propostas. Mesmo assim, se pôde comprovar que, ao responder a estas questôes, alguns estudantes também desprezaram os pontos de vista éticos dos seus colegas que entravam em conflito com os próprios. Outro estudo muito recente de Sadler, Chambers e Zeidler (2004) apoiou a idéia de que, para tomar decisóes sociocientíficas, muitos estudantes têm mais confiança na informação que é relevante para as suas crenças pessoais do que na qualidade científica das provas e dos dados proporcionados; ou seja, para eles não há uma relação direta entre a capacidade de persuasão dos dados e o seu valor científico.

Por outro lado, Bell e Lederman (2003) estudaram o papel das crenças sobre NdC, assim como as estratégias, pensamentos e fatores que mais influenciam na tomada de decisões sobre assuntos tecnocientíficos problemáticos. $\mathrm{O}$ resultado foi que os diferentes pontos de vista da $\mathrm{NdC}$ não são um fator crucial para tomar uma decisão sobre os dilemas tecnocientíficos propostos, pois o seu papel foi nulo para a maioria dos professores universitários que interviram no estudo e insignificante para o resto dos professores. O procedimento seguido pelos participantes na investigação para tomar estas decisões foi bastante semelhante na maioria dos casos, independentemente de seus pontos de vista sobre a $\mathrm{NdC}$ serem ou não adequados. Ainda que haja pequenas diferenças no pensamento que empregaram, as suas conseqüências foram escassas porque as decisões tomadas não foram suficientemente diferentes entre si. Para chegar a conclusões tão parecidas, provavelmente os participantes basearam-se em outros fatores diferentes das suas crenças sobre a NdC. De modo consistente com investigações anteriores sobre a tomada de decisões em questões sociocientíficas (Fleming, 1986a, b; Pedretti, 1999; Zeidler e Schafer, 1984), os fatores mais influentes foram os valores morais e pessoais, assim como os aspectos culturais, sociais e políticos, relacionados com as questôes colocadas.

O resultado desta investigação, que deriva de uma tese de doutorado (Bell, 1999), não deveria surpreender muito, pois já se sabe que, na prática científica, os dilemas tecnocientíficos e as controvérsias quase nunca se decidem apenas pelos dados disponíveis (Driver et al., 1996; Désautels e Larochelle, 2003). Com efeito, há muito tempo que um trabalho pioneiro de Zeidler e Schafer (1984) proporcionou dados empíricos que mostravam a importância dos aspectos morais e emotivos na resolução de assuntos tecnocientíficos de interesse social e, ainda há pouco, Sadler (2003) ampliou estas sugestôes na sua tese de doutorado, em que estudou o papel das emoções na tomada de decisões sobre questões relacionadas com a engenharia genética. Nesta investigação com estudantes universitários mostra-se que as considerações morais, os sentimentos, as emoções e as intuições têm grande influência nas decisões que se tomam sobre estas questôes, ainda que também aflorem outros fatores, como falta de informação sobre o tema colocado, experiências pessoais, crenças religiosas, família e cultura popular (Sadler e Zeidler, 2004).

A tomada de decisões é, sem dúvida, uma importante capacidade que pode ser educada. De fato, é um dos objetivos de muitos currículos de ciências que pode ser formulado de diversas maneiras, tais como o papel da ciência e da tecnologia na tomada de decisóes na comunidade, no trabalho, em casa etc. A participação na tomada de decisões pode colocar-se nas aulas de ciências para se trabalhar em grupo e tem já uma larga tradição tanto no trabalho experimental ou prático como em outros trabalhos de aula em equipe, especialmente na educação 
ambiental (Zoller, 1989). Contudo, para a abordar adequadamente parece necessário prestar muito mais atenção, do que habitualmente se faz em educação científica, aos aspectos culturais, sociais, morais e emotivos (Zeidler, Sadler e Simmons, 2003) e aos atitudinais e axiológicos, tal e como se vem propagando há muito tempo no movimento CTS para o ensino das ciências que pretende educar para a participação dos cidadãos nos assuntos tecnocientíficos de interesse social (Acevedo, 1996a; Acevedo, Vázquez e Manassero, 2002; Martín-Gordillo, 2003; MartínGordillo e Osorio, 2003; Martín-Gordillo, Osorio e López-Cerezo, 2001; Waks, 1996).

Nas últimas décadas os processos de tomada de decisōes em situações de controvérsia têm sido muito estudados pela psicologia tanto no caso de indivíduos como de grupos (León, 1994). Estas investigações têm-se desenvolvido como um campo de estudos independentes, mas os seus resultados poderiam proporcionar também valiosas pistas para educar a tomada de decisões no ensino das ciências. Em síntese, pode-se dizer que estes trabalhos têm mostrado que as decisões estão sempre enviesadas; isto é, apresentam um desvio sistemático em relação à solução que se considera mais racional. Por exemplo, demonstrou-se que os indivíduos tomam as suas decisões com grande quantidade de enviesamentos atribuídos ao uso de heurísticas que simplificam os processos implicados. Por isso se fala de uma racionalidade limitada que é determinada, entre outros fatores, pela percepção seletiva dos dados e pela dependência do contexto da decisão.

$\mathrm{Na}$ hora de tomar decisōes, os grupos e as comunidades costumam cometer menos erros que os indivíduos, pela sua maior capacidade para comprovar e corrigir possíveis enviesamentos e outras falhas, pondo em marcha, para isso, os seus próprios mecanismos de avaliação e controle (Duffy, 1993). Assim, em geral, a tomada de decisões pelos grupos seria melhor que a dos indivíduos. Contudo, sabe-se pouco sobre como e quando os grupos comprovam os erros. Fato é que nem sempre o fazem e, em certas circunstâncias, podem cometer mais erros por vezes também mais graves - que os próprios indivíduos.

Quando os modelos partilhados pelos membros do grupo alcançam um certo grau de homogeneidade que se reduz drasticamente a crítica interna, perde-se criatividade e originalidade nas contribuiçōes individuais e produz-se o fenômeno da tendência para a conformidade com a média do grupo. Além disso, se o grupo antepõe a sua própria identidade e o seu desejo de unanimidade, sobrepondo-se a outros pontos de vista mais plurais, chega-se ao denominado pensamento grupal, em que o grupo está tão coeso e o sentido de pertença a ele é tão forte que acaba produzindo uma visão demasiado homogênea dos problemas, da forma de abordá-los e de resolvêlos, caracterizada pela ilusão do consenso e por um excessivo otimismo (Janis, 1989).

\section{Natureza da Ciência e Didática das Ciências}

Os resultados dos estudos e investigações expostos neste trabalho deveriam fazer-nos refletir mais criticamente acerca das razões pelas quais há que incluir a $\mathrm{NdC}$ no ensino das ciências. Mas, além disso, no pressuposto de que há que o fazer, continuaria por resolver o problema de qual visão da $\mathrm{NdC}$ deve ser objeto da ciência escolar. Ainda que não seja possível fazer agora uma crítica bem fundamentada do modo como a Didática das Ciências se deve ocupar da $\mathrm{NdC}$ na ciência escolar, o que deveria ser objeto de um trabalho mais extenso e de outro tipo, parece, contudo, oportuno abordar aqui, pelo menos algumas reflexões sobre esta importante questão.

É fácil comprovar na bibliografia especializada que a Didática das Ciências presta atenção quase exclusiva aos traços característicos da natureza da ciência acadêmica dominante no passado, sem ter em conta que, desde o último quarto do século XX, uma boa parte da ciência é 
tecnociência e a sua natureza não responde aos mesmos padrões (Acevedo, 1997; Echeverría, 2003). De certo modo, está a reproduzir-se na Didática das Ciências uma situação semelhante a dos anos 50, 60 e 70, quando os filósofos da ciência discutiam sobre a $\mathrm{NdC}$ do passado, incluindo entre eles não só os representantes do positivismo lógico, mas também outros como Popper, Kuhn, Feyerabend, Lakatos, Laudan e todos os pensadores englobados no que se denominou nova filosofia da ciência - que agora é antiga. Ao que parece, estes debates faziamse sem ter em conta que, desde a Segunda Guerra Mundial, se não antes, a macrociência - big science - associada ao exército e às grandes indústrias já partilhava espaço com a ciência acadêmica de gabinete e de congressos (Echeverría, 2003). Gradualmente, depois, foi surgindo a tecnociência, que não é idêntica à macrociência, ainda que por vezes se confundam. Não é costume terem-se em conta estes fatos nos estudos de $\mathrm{NdC}$ que se desenvolvem em Didática das Ciências.

Certamente que, hoje em dia, ainda há muita ciência que não é macrociência nem tecnociência, do mesmo modo que também há tecnologia que não é alta tecnologia - high technology - nem tecnociência, e aí estão, todas elas, convivendo no presente. Contudo, a complexidade e o caráter poliédrico da cada vez mais poderosa tecnociência contemporânea é outro fator de dificuldade para o ensino da $\mathrm{NdC}$, que se soma a outras, como pode ser a busca de consenso sobre a $\mathrm{NdC}$. Deste modo, é bastante ingênuo pensar na existência de uma única $\mathrm{NdC}$, pelo que qualquer descrição da $\mathrm{NdC}$, só pode aspirar a refletir de maneira parcial e enviesada a ciência e a tecnociência do presente.

Em Didática das Ciências é necessário dar respostas a perguntas como as seguintes: a que tipo de ciência nos referimos quando falamos de $\mathrm{NdC}$ ?, qual é a $\mathrm{NdC}$ que pretendemos transmitir?, que consensos sobre a $\mathrm{NdC}$ podem ser válidos? E, sobretudo, para que queremos ensinar $\mathrm{NdC}$ ? Em particular, em relação a esta última questão, as propostas de $\mathrm{NdC}$ no ensino das ciências poderiam resultar estéreis sem ter em conta as finalidades da educação científica e para que deve ser relevante a ciência escolar (Acevedo, 2004). Por exemplo, se o que se pretende é educar para tomar melhores decisões cívicas no mundo atual - educação para a participação na cidadania na sociedade civil -, possivelmente é mais importante conhecer os aspectos da natureza da tecnociência que os da ciência acadêmica, pois, sem dúvida, a primeira é a que mais afeta a sociedade; para pequenas decisões da vida cotidiana, certamente é essencial uma melhor compreensão da natureza correspondente à tecnologia do tipo menos sofisticado; para satisfazer necessidades estéticas pessoais, poderia ser mais interessante o conhecimento da natureza da ciência acadêmica... Poucas propostas sobre estas questôes costumam aparecer nos artigos de Didática das Ciências a ponto de delimitar o problema da transposição didática da $\mathrm{NdC}$ para a ciência escolar.

\section{Conclusão}

Tal como ocorre no ensino das ciências, em certas ocasiões, a Didática das Ciências trasmite como mitos crenças que não estão suficientemente sustentadas pela investigação que ela própria produz; isto é, o faz sem cumprir os critérios de racionalidade científica aos quais costuma apelar. Também neste caso, os motivos para tomar as decisões parecem basear-se mais em outros fatores que se relacionam com o ideológico, o axiológico e o atitudinal, tais como as crenças e os valores culturais, pessoais e sociais. Assim, pois, os especialistas em Didática das Ciências muitas vezes tomam decisões no seu próprio campo de especialidade de modo semelhante ao que faz qualquer cidadão em outros assuntos, utilizando critérios que vão mais além dos invocados como científicos. 
Hoje em dia, o sistema de valores maioritariamente partilhado na Didática das Ciências faz-nos crer, a muitos de nós, que é bom ensinar algo da $\mathrm{NdC}$ e apontamos razões para justificar esta decisão. Não obstante, freqüentemente se ignoram as provas científicas que a investigação desenvolvida pela própria Didática das Ciências proporciona. Deveríamos ser conscientes de que em tal caso atuamos à margem de uma suposta racionalidade científica; de outra forma, deveríamos admitir que tomamos muitas decisões com critérios semelhantes aos que guiam a racionalidade leiga da cidadania. Sendo que os membros da comunidade de especialistas em Didática das Ciências constituem um conjunto de pessoas, que é relativamente numeroso no presente, talvez também se pudesse aplicar a esta comunidade de especialistas alguns dos resultados das investigações sobre a tomada de decisōes que se expuseram anteriormente. Sem dúvida, isto teria que nos fazer refletir um pouco mais acerca das críticas que fazemos ao ensino das ciências, aos professores e aos alunos e, em geral, à cidadania em relação à alfabetização científica e tecnológica, assim como sobre as propostas que fazemos e as contradiçōes que temos.

\section{Referências}

AMERICAN ASSOCIATION FOR THE ADVANCEMENT OF SCIENCE. Science for all americans. New York: Oxford University Press, 1989.

. Benchmarks for science literacy: a project 2061 report. New York: Oxford University Press, 1993.

ABD-EL-KHALICK, F. Embedding nature of science instruction in preservice elementary science:

abandoning scientism, but... Journal of Science Teacher Education, Toledo, v. 12, n. 3, p. 215-233, 2001.

. The influence of a philosophy of science course on preservice secondary science teachers'

views of nature of science. In: ANNUAL INTERNATIONAL CONFERENCE OF THE

ASSOCIATION FOR THE EDUCATION OF TEACHERS IN SCIENCE, 2002, Pensacola.

Proceedings... Pensacola: AETS, 2002.

Disponível em: http://www.ed.psu.edu/CI/Journals/2002aets/f7_abd_el_khalick.rtf. Acesso em 7 jul. 2005.

ABD-EL-KHALICK, F.; BELL, R. L.; LEDERMAN, N. G. The nature of science and instructional practice: making the unnatural natural. Science Education, New York, v. 82, n. 4, p. 417-436, 1998.

ABD-EL-KHALICK, F.; BOUJAOUDE, S. An exploratory study of the knowledge base for science teaching. Journal of Research in Science Teaching, New York, v. 34, n. 7, p. 673-699, 1997.

ABD-EL-KHALICK, F.; LEDERMAN, N. G. Improving science teachers' conceptions of nature of science: a critical review of the literature. International Journal of Science Education, London, v. 22, n. 7, p. 665-701, 2000a.

. The influence of history of science course on students' views of nature of science. Journal of Research in Science Teaching, New York, v. 37, n. 10, p. 1057-1095, 2000 b.

ACEVEDO DÍAZ, J. A. Los futuros profesores de enseñanza secundaria ante la sociología y la epistemología de las ciencias. Revista Interuniversitaria de Formación del Profesorado, Zaragoza, v. 19, p. 111-125, 1994. Disponível em: http://www.campus-oei.org/salactsi/acevedo8.htm. Acesso em 7 jul. 2005. 
- Cambiando la práctica docente en la enseñanza de las ciencias a través de CTS. Sala de

Lecturas CTS+I de la OEI. 2001.

Disponível em: http://www.campus-oei.org/salactsi/acevedo2.htm. Acesso em 7 jul. 2005.

ACEVEDO DÍAZ, J. A. La formación del profesorado de enseñanza secundaria y la educación CTS. Una cuestión problemática. Revista Interuniversitaria de Formación del Profesorado, Zaragoza, v. 26, p. 131-144, 1996b.

Disponível em: http://www.campus-oei.org/salactsi/acevedo9.htm. Acesso em 7 jul. 2005.

- ¿Publicar o patentar? Hacia una ciencia cada vez más ligada a la tecnología. Revista Española de Física, Madrid, v. 11, n. 2, p. 8-11, 1997.

Disponível em: http://www.campus-oei.org/salactsi/acevedo4.htm. Acesso em 7 jul. 2005.

Reflexiones sobre las finalidades de la enseñanza de las ciencias: educación científica para la ciudadanía. Revista Eureka sobre Enseñanza y Divulgación de las Ciencias, Cádiz, v. 1, n. 1, p. 3-16, 2004. Disponível em: http://www.apac-eureka.org/revista/Larevista.htm. Acesso em 7 jul. 2005.

ACEVEDO DÍAZ, J. A.; VÁZQUEZ ALONSO, A.; MANASSERO MAS, M. A. El movimiento Ciencia, tecnología y sociedad y la enseñanza de las ciencias. Sala de Lecturas CTS+I de la OEI, 2002. Disponível em: http://www.campus-oei.org/salactsi/acevedo13.htm>. Acesso em 7 jul. 2005.

ACEVEDO DÍAZ, J. A.; ACEVEDO ROMERO, P. Proyectos y materiales curriculares para la educación CTS: enfoques, estructuras, contenidos y ejemplos. Bordón, Madrid, v. 54, n.1, p. 5-18, 2002. Disponível em: http://www.campus-oei.org/salactsi/acevedo19.htm. Acesso em 7 jul. 2005.

ADÚRIZ-BRAVO, A. Integración de la epistemología en la formación del profesorado de ciencias. 2001. 622 f. Tesis (Doctoral en Ciencias Sociales) - Departament de Didàctica de les Matemàtiques i de les Ciències Experimentals, Universitat Autònoma de Barcelona, Bellaterra, 2001.

AKERSON, L.; ABD-EL-KHALICK, F.; LEDERMAN, N. G. Influence of a reflective explicit activity-based approach on elementary teachers' conceptions of nature of science. Journal of Research in Science Teaching, New York, v. 37, n. 4, p. 295-317, 2000.

ALTERS, B.J. Whose nature of science? Journal of Research in Science Teaching, New York, v. 34, n. 1, p. 39-55, 1997.

BARTHOLOMEW, H.; OSBORNE, J.; RATCLIFFE, M. Teaching pupils 'ideas-about-science’: case studies from the classroom. In: ANNUAL MEETING OF THE NATIONAL ASSOCIATION FOR RESEARCH IN SCIENCE TEACHING, 2002, New Orleans. Paper... New Orleans: [s.n], 2002. Disponível em: http://www.york.ac.uk/depts/educ/projs/NARST2002_P3. Acesso em 7 jul. 2005.

BELL, R. L. Understanding of the nature of science and decision making on science and technology based issues. Dissertation Abstract International, v. 60, p. 3310, 1999.

BELL, R. L.; LEDERMAN, N. G. Understandings of the nature of science and decision making on science and technology based issues. Science Education, New York, v. 87, n. 3, p. 352-377, 2003. 


\section{J. A. Acevedo, A. Vásquez, M. F. Paixão, P. Acevedo, J. M. Oliva e M. A. Manassero}

BELL, R. L.; LEDERMAN, N. G.; ABD-EL-KHALICK, F. Implicit versus explicit nature of science instruction: an explicit response to Palmquist and Finley. Journal of Research in Science Teaching, New York, v. 35, n. 9, p. 1057-1061, 1998.

BELL, R. L.; LEDERMAN, N. G.; ABD-EL-KHALICK, F. Developing and acting upon one's conception of the nature of science: a follow-up study. Journal of Research in Science Teaching, New York, v. 37, n. 6, p. 563-581, 2000.

BYBEE, R. W. Achieving scientific literacy: from purposes to practices. Portsmouth: Heinemann, 1997.

CAAMAÑO, A. La comprensión de la naturaleza de la ciencia: un objetivo de la enseñanza de las ciencias en la ESO. Alambique, v. 8, n. 43-51, 1996.

CANAVARRO, J. M. O que se pensa sobre a ciência. Coimbra: Quarteto, 2000.

CARVAJAL, E.; GÓMEZ-VILLARTA, M. R. Concepciones y representaciones de los maestros de secundaria y bachillerato sobre la naturaleza, el aprendizaje y la enseñanza de las ciencias. Revista Mexicana de Investigación Educativa, Tlalpan, v. 7, n. 16, p. 577-602, 2002.

SPAIN. Decreto 106/1992, de 9 de junio, por el que se establecen las enseñanzas correspondientes a la educación secundaria obligatoria en Andalucía. Boletín Oficial de la Junta de Andalucía, Sevilla, 56, 1992.

. Decreto 148/2002, de 14 de mayo, por el que se modifica el Decreto 106/1992, de 9 de junio, por el que se establecen las enseñanzas correspondientes a la educación secundaria obligatoria en Andalucía. Boletín Oficial de la Junta de Andalucía, Sevilla, 75, 2002.

DEPARTMENT FOR EDUCATION AND EMPLOYMENT. Science in the National Curriculum. London: HMSO, 1999.

DÉSAUTELS, J.; LAROCHELLE, M. Educación científica: el regreso del ciudadano y de la ciudadana. Enseñanza de las Ciencias, Barcelona, v. 21, n. 1, p. 3-20, 2003.

DRIVER, R. et al. Young people’s images of science. Buckingham: Open University Press, 1996.

DUFFY, L. Team decision making and technology. In: CASTELLAN Jr., N. J. (Ed.). Individual and group decision making: current issues. Hillsdale: LEA, 1993. p. 247-266.

DUSCHL, R. A.; WRIGHT, E. A case study of high school teachers' decision making models for planning and teaching science. Journal of Research in Science Teaching, New York, v. 26, n. 6, p. 467-501, 1989.

ECHEVERRÍA, J. La revolución tecnocientífica. Madrid: FCE, 2003.

EFLIN, J. T.; GLENNAN, S.; REISCH, R. The nature of science: a perspective from the philosophy of science. Journal of Research in Science Teaching, New York, v. 36, n. 1, p. 107-116, 1999. 
FELSKE, D. D.; CHIAPPETTA, E.; KEMPER, J. A historical examination of the nature of science and its consensus in benchmarks and standards. In: ANNUAL MEETING OF THE NATIONAL ASSOCIATION FOR RESEARCH IN SCIENCE TEACHING, 2001, Saint Louis. Paper... Saint Louis: [s.n.], 2001.

FERNÁNDEZ-MONTORO, I. Análisis de las concepciones docentes sobre la actividad científica: una propuesta de transformación. 2000. Tesis (Doctoral) - Departamento de Didáctica de las Ciencias Experimentales y Sociales, Universitat de València, València, 2000.

FLEMING, R.W. Adolescent reasoning in socio-scientific issues, part I: social cognition. Journal of Research in Science Teaching, New York, v. 23, n. 8, p. 677-687, 1986a.

. Adolescent reasoning in socio-scientific issues, part II: non-social cognition. Journal of Research in Science Teaching, New York, v. 23, n. 8, p. 689-698, 1986 b.

GESS-NEWSOME, J.; LEDERMAN, N. G. Examining pedagogical content knowledge: the construct and its implications for science education. Dordrecht: Kluwer Academic Press, 1999.

GROSS, P.; LEVITT, N. Higher superstition: the academic left and its quarrels with science. Baltimore: John Hopkins University Press, 1994.

HODSON, D. Hacia un enfoque más crítico del trabajo de laboratorio. Enseñanza de las Ciencias, Barcelona, v. 12, n. 3, p. 299-313, 1994.

IRWIN, A. R. Historical case studies: teaching the nature of science in context. Science Education, New York, v. 84, n. 1, p. 5-26, 2000.

JANIS, I. L. Crucial decision making. New York: The Free Press, 1989.

KHISHFE, R.; ABD-EL-KHALICK, F. Influence of explicit reflective versus implicit inquiry-oriented instruction on sixth graders' views of nature of science. Journal of Research in Science Teaching, New York, v. 39, n. 7, p. 551-581, 2002.

KOLSTØ, S.D. Consensus projects: teaching science for citizenship. International Journal of Science Education, London, v. 22, n. 6, p. 645-664, 2000.

. Scientific literacy for citizenship: tools for dealing with the science dimension of controversial socioscientific issues. Science Education, New York, v. 85, n. 3, p. 291-310, 2001.

KOLSTØ, S. D. et al. Science student' critical assessment of scientific information related to socioscientific controversies. In: ANNUAL MEETING OF THE NATIONAL ASSOCIATION FOR RESEARCH IN SCIENCE TEACHING, 2004, Vancouver. Paper... Disponível em: http://www.uib.no/people/pprsk/Dankert/Handouts/Handouts. Acesso em 7 jul. 2005. 
KOLSTØ, S. D.; MESTAD, I. Learning about the nature of scientific knowledge: the imitating science project. In: CONFERENCE OF THE EUROPEAN SCIENCE EDUCATION RESEARCH ASSOCIATION (ESERA), 4, 2003, Noordwijkerhout. Research and the Quality of Science Education. Disponível em: resumo http://www1.phys.uu.nl/esera2003/program.shtml, texto completo http://www.uib.no/people/pprsk/Dankert/Handouts/Handouts. Acesso em 7 jul. 2005.

LEDERMAN, N. G. Students' and teachers' conceptions of the nature of science: a review of the research. Journal of Research in Science Teaching, New York, v. 29, n. 4, p. 331-359, 1992.

. Teachers' understanding of the nature of science: factors that facilitate or impede the relationship. Journal of Research in Science Teaching, New York, v. 36, n. 8, p. 916-929, 1999.

LEDERMAN, N. G.; MCCOMAS, W. F.; MATTHEWS, M. R. (Ed.). Science \& Education, Dordrecht, v. 7, n. 6, p. 507-509, 1998.

LEDERMAN, N. G.; ZEIDLER, D. Science teachers' conceptions of the nature of science: do they really influence teaching behavior? Science Education, New York, v. 71, n. 5, p. 721-734, 1987.

LEÓN, O. G. Análisis de decisiones. Madrid: McGraw-Hill, 1994.

LIN, H. S.; CHEN, C. C. Promoting preservice chemistry teachers' understanding about the nature of science through history. Journal of Research in Science Teaching, New York, v. 39, n. 9, p. 773-792, 2002.

MANASSERO, M. A., VÁZQUEZ, A.; ACEVEDO, J. A. Evidences for consensus on the nature of science issues. In: JANIUK, R. M.; SAMONEK-MICIUK, E. (Ed.). Science and technology education for a diverse world: dilemmas, needs and partnerships. Lublin: Marie Curie-Sklodowska University Press, 2004. p. 167-168.

MARTÍN-GORDILLO, M. Metáforas y simulaciones: alternativas para la didáctica y la enseñanza de las ciencias. Revista Electrónica de Enseñanza de las Ciencias, v. 2, 2003.

Disponível em: http://www.saum.uvigo.es/reec/. Acesso em 7 jul. 2005.

MARTÍN-GORDILLO, M.; OSORIO, C. Educar para participar en ciencia y tecnología. Un proyecto para la difusión de la cultura científica. Revista Iberoamericana de Educación, v. 32, p. 165-210, 2003. Disponível em: http://www.campus-oei.org/revista/rie32a08.PDF. Acesso em 7 jul. 2005.

MARTÍN-GORDILLO, M.; OSORIO, C.; LÓPEZ-CEREZO, J. A. La educación en valores a través de CTS. In: FORO IBEROAMERICANO SOBRE EDUCACIÓN EN VALORES, 2000, Madrid. La educación en valores en Iberoamérica. Madrid: OEI, 2001. p. 119-161.

Disponível em: www.campus-oei.org/salactsi/mgordillo.htm. Acesso em 7 jul. 2005.

MATKINS, J. J. et al. Impacts of contextual and explicit instruction on preservice elementary teachers understandings of the nature of science. In: ANNUAL INTERNATIONAL CONFERENCE OF THE ASSOCIATION FOR THE EDUCATION OF TEACHERS IN SCIENCE, 2002, Pensacola. Proceedings... Pensacola: AETS, 2002. p. 456-481.

Disponível em: http://www.ed.psu.edu/CI/Journals/2002aets/s2_matkins_bell_irving_m.rtf. Acesso em 7 jul. 2005. 
MATTHEWS, M. R. In defense of modest goals when teaching about the nature of science. Journal of Research in Science Teaching, New York, v. 35, n. 2, p. 161-174, 1998a.

. The nature of science and science teaching. In: FRASER, B. J.; TOBIN, K. G. (Ed.). International handbook of science education. Dordrecht: Kluwer Academic Publishers, 1998b. p. 981-999.

MCCOMAS, W. F., CLOUGH, M. P.; ALMAZROA, H. The role and character of the nature of science in science education. In: McCOMAS, W. F. (Ed.). The nature of science in science education: rationales and strategies. Dordrecht: Kluwer Academic Publishers, 1998. p. 3-39.

McCOMAS, W. F.; OLSON, J. K. The nature of science in international science education standards documents. In: McCOMAS, W. F. (Ed.). The nature of science in science education: rationales and strategies. Dordrecht: Kluwer Academic Publishers, 1998. p. 41-52.

MELLADO, V. Concepciones y prácticas de aula de profesores de ciencias, en formación inicial de primaria y secundaria. Enseñanza de las Ciencias, Barcelona, v. 14, n. 3, p. 289-302, 1997.

MELLADO, V. Preservice teachers' classroom practice and their conceptions of the nature of science. Science \& Education, Dordrecht, v. 6, n. 4, p. 331-354, 1997.

MELLADO, V. Preservice teachers' classroom practice and their conceptions of the nature of science. In: FRASER, B. J.; TOBIN, K. G. (Ed.). International handbook of science education. Dordrecht: Kluwer Academic Publishers, 1998. p. 1093-1110.

. The classroom practice of preservice teachers and their conceptions of teaching and learning science. Science Education, New York, v. 82, n. 2, p. 197-214, 1998.

NATIONAL RESEARCH COUNCIL. National science education standards. Washington: National Academic Press, 1996.

OSBORNE, J. et al. What 'ideas-about-science' should be taught in school science?: a Delphi study of the expert community. Journal of Research in Science Teaching, New York, v. 40, n. 7, p. 692-720, 2003.

. What should we teach about science: a Delphi study, evidence-based practice in science education (EPSE) report, school of education. London: King's College, 2001.

Disponível em: http://www.york.ac.uk/depts/educ/projs/DelphiReport. Acesso em 7 jul. 2005.

PEDRETTI, E. Decision making and STS education: exploring scientific knowledge and social responsibility in schools and science centers through an issues-based approach. School Science and Mathematics, Menasha, v. 99, p. 174-181, 1999.

REID, D. J.; HODSON, D. Ciencia para todos en secundaria. Traducção de M. J. Martín-Díaz e L. A. García-Lucía. Madrid: Narcea, 1993.

RUDOLPH, J. L. Portraying epistemology: school science in historical context. Science Education, New York, v. 87, n. 1, p. 64-79, 2003. 


\section{J. A. Acevedo, A. Vásquez, M. F. Paixão, P. Acevedo, J. M. Oliva e M. A. Manassero}

SADLER, T. D. Informal reasoning regarding socioscientific issues: the influence of morality and content knowledge. 2003. Tesis (Doctoral) - University of South Florida, Tampa, 2003.

SADLER, T. D.; CHAMBERS, W. F.; ZEIDLER, D. Student conceptualizations of the nature of science in response to a socioscientific issue. International Journal of Science Education, London, v. 26 , n. 4 , p. $387-409,2004$.

SADLER, T. D.; ZEIDLER, D. The morality of socioscientific issues: construal and resolution of genetic engineering dilemmas. Science Education, New York, v. 88, n. 1, p. 4-27, 2004.

SCHWARTZ, R. S.; LEDERMAN, N. G. "It's the nature of the beast": the influence of knowledge and intentions on learning and teaching nature of science. Journal of Research in Science Teaching, New York, v. 39, n. 3, p. 205-236, 2002.

SCHWARTZ, R. et al. Explicit/reflective instructional attention to nature of science and scientific inquiry: impact on student learning. In: ANNUAL INTERNATIONAL CONFERENCE OF THE ASSOCIATION FOR THE EDUCATION OF TEACHERS IN SCIENCE, 2002, Pensacola.

Proceedings... Pensacola: AETS, 2002. p. 646-667.

Disponível em: http://www.ed.psu.edu/CI/Journals/2002aets/f5_schwartz_lederman_k.rtf. Acesso em 7 jul. 2005.

SHULMAN, L. Those who understand: knowledge growth in teaching. Educational Researcher, Washington, v. 15, n. 2, p. 4-14, 1986.

SHULMAN, L. Knowledge and teaching: foundations of the new reform. Harvard Educational Review, Cambridge, v. 57, p. 1-22, 1987.

SMITH, M. U. et al. How great is the disagreement about the nature of science?: a response to alters. Journal of Research in Science Teaching, New York, v. 34, n. 10, p. 1101-1104, 1997.

SOKAL, A.; BRICMONT, J. Intellectual impostures: postmodern philosophers' abuse of science. London: Profile, 1998.

Imposturas intelectuales. Traducção de J.C. Guix Vilaplana. Barcelona: Paidós, 1999.

SPECTOR, B.; STRONG, P.; LAPORTA, T. Teaching the nature of science as an element of science, technology and society. In: McCOMAS, W. F. (Ed). The nature of science in science education: rationales and strategies. Dordrecht: Kluwer Academic Publishers, 1998. p. 267-276.

TSAI, C. C. Nested epistemologies: science teachers' beliefs of teaching, learning and science. International Journal of Science Education, London, v. 24, n. 8, p. 771-783, 2002.

VÁZQUEZ, A.; ACEVEDO, J. A.; MANASSERO, M. A. Consensos sobre la naturaleza de la ciencia: evidencias e implicaciones para su enseñanza. In: Revista Iberoamericana de Educación, Madrid, 2004. Disponible em: http://www.campus-oei.org/revista/deloslectores/702Vazquez.PDF. Acesso em 7 jul. 2005. 
VÁZQUEZ, A. et al. Cuatro paradigmas básicos sobre la naturaleza de la ciencia. Argumentos de Razón Técnica, Sevilla, v. 4, p. 135-176, 2001.

Disponible em: http://www.campus-oei.org/salactsi/acevedo20.htm. Acesso em 7 jul. 2005.

. Hacia un consenso sobre la naturaleza de la ciencia en la enseñanza de las ciencias. In: MARTINS, I. P.; PAIXÃO, F.; VIEIRA, R. (Org.). Perspectivas ciência-tecnologia-sociedade na inovação da educação em ciência. Aveiro: Universidade de Aveiro, 2004. p. 129-132.

WAKS, L. J. Las relaciones escuela-comunidad y su influencia en la educación en valores en CTS. In: ALONSO, A.; AYESTARÁN, I.; URSÚA, N. (Ed.). Para comprender ciencia, tecnología y sociedad. Estella: EVD, 1996. p. 35-47.

WOLPERT, L. The Unnatural Nature of Science. London: Faber \& Faber, 1992.

. La naturaleza no natural de la ciencia. Madrid: Acento Editorial, 1994.

ZEIDLER, D. L.; SADLER, T. D.; SIMMONS, M. L. Morality and socioscientific issues in science education: current research and practice. In: CONFERENCE OF THE EUROPEAN SCIENCE EDUCATION RESEARCH ASSOCIATION (ESERA), 4, 2003. Noordwijkrhout. Research and the quality of science education.

Disponível em: http://www1.phys.uu.nl/esera2003/program.shtml. Acesso em 7 jul. 2005.

ZEIDLER, D. L.; SCHAFER, L. E. Identifying meditating factors of moral reasoning in science education. Journal of Research in Science Teaching, New York, v. 21, n. 1, p. 1-15, 1984.

ZEIDLER, D. L. et al. Tangled up in views: beliefs in the nature of science and responses to socioscientific dilemmas. Science Education, New York, v. 86, n. 3, p. 343-367, 2002.

ZOLLER, U. Critical and system thinking, problem solving and decision making in STES (Science-Technology-Environment-Society) education. In: GROSS, M. G. et al. (Ed.). Working together to educate about the environment. Troy: NAEE/CEA, 1989. p. 371-377. 
